\title{
Substantiating parameters brake system of the tractor trailer
}

\author{
Abdulaziz Shermukhamedov ${ }^{*}$, Nurillo Ergashev, and Abdukhamid Azizov \\ Tashkent state transport university, Tashkent, Uzbekistan
}

\begin{abstract}
The article discusses substantiating the parameters brake system of a tractor-trailer (TT). The section offers a comparative analysis of theoretical and experimental studies of the TT brake drive and the parameters of its elements. Based on that, ordinary differential equations were solved by the Runge - Kutta method, the first-order accuracy (Euler's method). To solve partial differential equations, we used a modified Lax Wendroff scheme. The results were obtained using the methods described above are theoretically very consistent with the triggering time $t_{s}=0.47 \mathrm{~s}$ and the experimental value $0.46 \mathrm{~s}$. Thus, the studying dynamic circuits of the pneumatic drive of TT brakes showed a high converging theoretical characteristic for a typical control line of a drive with an accelerating valve with experimental data, and the error was no more than $5 \%$.
\end{abstract}

\section{Introduction}

As the mainstay of the world's economy, the vehicle is also one of the most important transportations for human beings $[1,3]$. However, the main important factor for human life and quality delivery of cargo using vehicles is the braking system. Therefore, the substantiation brake parameters of the vehicles remain one of the key issues.

In carrying out this work, in the braking system design of a vehicle, the first consideration should be the braking performance. The braking system has to be able to stop or reduce the vehicle speed as quickly as possible and maintain the vehicle travelling direction in a stable and controllable manner in any road condition $[4,8]$. The stability of heavy vehicles, a particularly articulated vehicle, is a very important and complex issue. Tractor/trailer systems can be inherently unstable in certain conditions, such as high-speed hard braking. In such conditions, stability is not always maintained by passive braking systems, even if they are in perfect operating condition [6]. The disadvantages of the conventional pneumatic brake systems, such as response delay and unreasonable braking force distribution, may cause serious accidents $[9,10]$.

\section{Methods}

Given that tractor trailers' carrying capacity and speed often depend on the condition of their brake systems, the braking speed and synchronous operation of all trailers are also

\footnotetext{
* Corresponding author: sheraziz@mail.ru
} 
important. Therefore, it is necessary to create such a brake actuator that ensures efficient and stable operation of the TT during braking, regardless of where the trailers are installed in the TT [11].

For this purpose, it is developed, patented, and introduced into high-speed production pneumatic and electro-pneumatic brake drives for TT $[12,13]$, which provide standard speed and synchronous operation of the brakes of all train links.

The section offers a comparative analysis of theoretical and experimental studies of the TT brake drive and the parameters of its elements.

In theoretical studies, a mathematical model is presented for the high-precision calculation of the operation of the TT brake drive and the parameters of its elements.

Figure 1 shows a diagram of the pneumatic drive of the braking system of a tractor trailer, which consists of sequentially connected independent sections: section $l$ [brake crane $(\mathrm{BC})$ is pipeline $l_{l}$ - accelerating valve (AV)]; section $2\left[\mathrm{AV}\right.$ - pipeline $l_{2}$ - air distribution valve (ADV)]; section $3\left[\mathrm{ADV}\right.$ - pipelines $l_{3} \div l_{8}$ - wheel brake chambers (WBCh)].

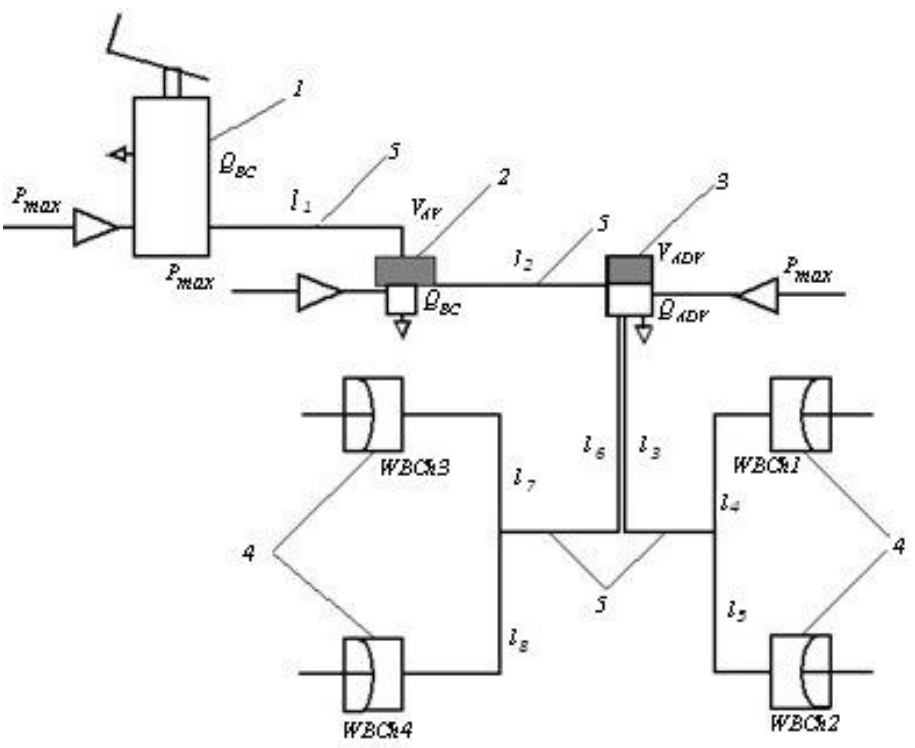

Fig. 1. Diagram of the pneumatic drive of the brakes of an auto-tractor trailer: 1 is $B C ; 2$ is AV; 3 is $\mathrm{ADV} ; 4$ is $\mathrm{WBCh} ; 5$ are pipelines

The mathematical model is built on a modular basis, that is, for each element of the pneumatic drive - its own model and their connection according to the adopted scheme and boundary conditions. This principle makes it possible to significantly reduce the time spent on compiling and implementing the calculation program on a computer [14].

The brake crane is a follower and represents the local resistance. The flow rate through such local resistance is expressed by the relationship [15]:

$$
Q_{B C}=\mu_{B C} f_{B C} \sqrt{\frac{2}{\rho}\left(p_{\text {in }}-p_{\text {out }}\right)}
$$

where $Q_{B C}$ is the flow rate at the outlet of the $\mathrm{BC} ; \mu_{B C}$ is consumption coefficient; $f_{B C}$ is flow area of the $\mathrm{BC} ; \rho$ is air density; $p_{\text {in }}$ and $p_{\text {out }}$ is pressure at the input and output of the $\mathrm{BC}$. 
The flow area of the $\mathrm{BC}$ is determined by the formula

$$
f_{B C}=\pi * d_{I V} * h_{I V}
$$

where $d_{I V}$ is the diameter of the input valve seat (IV); $h_{I V}$ - IV stroke:

$$
h_{I V}=\left\{\begin{array}{l}
0 \text { at } 0<t \leq \tau_{0} \\
h_{0} t /\left(i_{\mathrm{n}} t_{\max }\right) \quad \text { at } \quad \tau_{0}<t \leq \tau_{0}+t_{\max } \\
h_{0} / i_{\mathrm{n}} \text { at } t>\tau_{0}+t_{\max }
\end{array}\right.
$$

Here $h_{0}$ is the maximum IV stroke; $i_{n}$ is gear ratio of the crane mechanical drive; $\tau_{0}$ is time of selection of gaps in the mechanical drive; $t_{\max }$ is brake pedal travel time.

Dynamic processes in pipelines with an arbitrary cross-sectional area can be described by the one-dimensional Navier - Stokes equation with the flow parameters averaged over the cross-section (pressure $p$, density $\rho$ and velocity $V$ ).

In this case, the viscosity is taken into account only at the interface between the flow and the inner wall of the pipeline as a loss of pressure due to friction, as well as between sections perpendicular to the $\mathrm{x}$-axis of the pipeline. The air flow, in this case, is described by the system of equations [16]:

$$
\begin{aligned}
& \frac{\partial(\rho V)}{\partial t}+\frac{\partial\left(\rho V^{2}\right)}{\partial x}+\frac{\lambda}{2 d} \rho V^{2} \operatorname{sign}(V)=-\frac{\partial}{\partial x}\left(p-\frac{4}{3} \mu \frac{\partial V}{\partial x}\right) \\
& f \frac{\partial p}{\partial t}+f k \frac{p}{\rho} \frac{\partial(\rho V)}{\partial x}+k p V \frac{\partial f}{\partial x}=0 \\
& \frac{\mathrm{d}(\ln p)}{\mathrm{d} t}=\left[k-\frac{4}{3}(k-1) \frac{\mu}{p} \frac{\partial V}{\partial x}\right] \frac{\mathrm{d}(\ln \rho)}{\mathrm{d} t}
\end{aligned}
$$

where $\lambda$ is the coefficient of hydraulic friction; $d$ and $f$ is diameter and cross-sectional area of the pipeline; $\mu$ is the dynamic coefficient of viscosity; $k$ is the adiabatic exponent.

When air moves through the pipelines of the TT brake system, steep frontal waves can arise, which complicates the calculation of such flows. In particular, during the numerical solution, a jump in the air parameters in the wave front can be observed, the cause of which is its low viscosity. In this regard, Neumann and Richtmeier proposed to increase the dynamic coefficient of viscosity in the wave front, artificially assuming.

$$
\mu=\mu_{0} \rho \frac{\partial V}{\partial x}
$$

where $\mu_{0}$ is a coefficient characterizing the amount of front "blurring". This hypothesis, which makes it possible to provide an end-to-end calculation of steep wave fronts, is the basis for calculating the TT pneumatic braking systems.

Let us denote $k \frac{p}{\rho}=a^{2} ; u=\rho V$ and write equations (2) - (4) taking into account equality (5) in the form: 


$$
\begin{gathered}
f \frac{\partial u}{\partial t}+f \frac{\partial(p+w)}{\partial x}+f \frac{\partial}{\partial x}\left(\frac{a^{2} u^{2}}{k p}\right)+\frac{a^{2} u^{2}}{k p} \frac{\partial f}{\partial x}+\frac{f \lambda a^{2} u^{2}}{2 d k p} \operatorname{sign}(u)=0 \\
f \frac{\partial p}{\partial t}+f a^{2} \frac{\partial u}{\partial x}+a^{2} u \frac{\partial f}{\partial x}=0 \\
\frac{\partial(\ln \rho)}{\partial t}=-V \frac{\partial(\ln \rho)}{\partial x}+\frac{1}{k+(k-1) w / \rho}\left[\frac{\partial(\ln p)}{\partial t}+V \frac{\partial(\ln p)}{\partial x}\right] \\
w=\left\{\begin{array}{l}
-\mu_{0} \rho\left(\frac{\partial V}{\partial x}\right)^{2}, \frac{\partial V}{\partial x}<0 \\
0, \frac{\partial V}{\partial x} \geq 0
\end{array}\right.
\end{gathered}
$$

where $w$ is artificial viscosity [17]; a is the speed of sound.

The analysis of the operation of the pneumatic drive of the TP brakes showed that the diameters of its pipelines during the transient process change insignificantly, and they can be considered constant, i.e., $f=$ const. In this case, the fourth term in equation (6) and the third term in equation (7) will be equal to zero. When using equation (8), it is important to take into account the air flow regime determined by the coefficient $\lambda$ of hydraulic friction, which depends on the dimensionless parameters: Reynolds number $R_{r}=V d / v$ and relative roughness $\varepsilon=k_{w} / d$ of the pipeline wall, where $k_{w}$ is the roughness coefficient. Therefore, $\lambda$ $=f\left(R_{r}, k_{w} / d\right)$.

The complex influence of the parameters $R_{e}$ and $\varepsilon$ of the mainline on the coefficient $\lambda$ with an accuracy sufficient for practical calculations can be taken into account if the dependence $\lambda=f\left(\mathrm{R}_{\mathrm{r}}, k_{\mathrm{w}} / d\right)$ is approximated by a hyperbolic function of the form [15].

$$
\lambda=\frac{70}{R_{r}}+k_{\varepsilon}=\frac{70 v}{V d}+k_{\varepsilon}
$$

where $k_{\varepsilon}$ is coefficient of approximation, depending on the relative roughness $\varepsilon$ of the walls of the main lines; $v$ is kinematic viscosity of the gas.

Below are the values of $k_{\varepsilon}$ depending on $\varepsilon$ :

\begin{tabular}{|c|c|c|c|c|c|}
\hline$\varepsilon$ & 0.0001 & 0.0001 & 0.002 & 0.005 & 0.010 \\
\hline$k_{\varepsilon}$ & 0.0186 & 0.022 & 0.026 & 0.031 & 0.038 \\
\hline
\end{tabular}

Expression (9) automatically considers the effect of the fluid flow regime on the coefficient of hydraulic friction.

Dividing equations (6) - (8) by $\mathrm{f}$ and substituting expression (9) into equation (6), we obtain a system of equations:

$$
\begin{aligned}
& \frac{\partial u}{\partial t}+\frac{\partial(p+w)}{\partial x}+\frac{\partial}{\partial x}\left(\frac{a^{2} u^{2}}{k p}\right)+\frac{35 v u}{d^{2}} \operatorname{sign}(u)+\frac{k_{\varepsilon} a^{2} u^{2}}{2 d k p} \operatorname{sign}(u)=0 \\
& \frac{\partial p}{\partial t}+a^{2} \frac{\partial u}{\partial x}=0
\end{aligned}
$$




$$
\frac{\partial(\ln \rho)}{\partial t}=-V \frac{\partial(\ln \rho)}{\partial x}+\frac{1}{k+(k-1) w / \rho}\left[\frac{\partial(\ln p)}{\partial t}+V \frac{\partial(\ln p)}{\partial x}\right]
$$

It is known that the pipeline has different local resistances (bends, turns, areas of expansion or contraction), which are characterized by their own pressure losses, determined by the expression [18]:

$$
p_{m}=\varsigma \frac{\rho}{2} V^{2}
$$

where $\zeta$ is the coefficient of local resistance, determined empirically.

In the engineering literature on hydraulics (pneumatics), there are tables of local resistances for different sections of pipelines and units. Often, local resistances are replaced by an additional line length $\left(l_{e}\right)$, equivalent to pressure losses and determined by the expression [19]:

$$
l_{e}=\varsigma d / \lambda
$$

In the general case, the dynamics of the $\mathrm{AV}, \mathrm{ADV}$, and $\mathrm{WBCh}$ are described by the same equations. Let us consider the dynamic characteristics of a container with variable (WBCh) and constant (AV, ADV) boundaries. Automotive and tractor pneumatic drives are generally fast-acting, so heat exchange between the air in the drive and the environment during the transient process can be neglected. In this case, the equation of state of air in the tank has the form [20]:

$$
k R T \mathrm{~d} m=k p_{a} \mathrm{~d} V_{a}+V_{a} \mathrm{~d} p_{a}
$$

where $\mathrm{R}=287.14$ is the gas constant for air, $\mathrm{m}^{2} /\left(\mathrm{s}^{2} \mathrm{~K}\right) ; \mathrm{T}=293$ - absolute air temperature in the tank, $K ; m$ is the mass of air in the container, $\mathrm{kg} ; V_{a}$ is the volume of air in the tank, $m^{3} ; p_{a}$ is pressure in the container, $N / m^{2}$.

After transformation, equation (14) takes the form:

$$
\frac{\mathrm{d} p_{a}}{\mathrm{~d} t}=\frac{k R T}{V_{a}} \frac{\mathrm{d} m}{\mathrm{~d} t}-\frac{k p_{a}}{V_{a}} \frac{\mathrm{d} V_{a}}{\mathrm{~d} t}
$$

If we take into account that $d m / d t=Q_{a} \rho$, then equation (15) can be written in the form:

$$
\frac{\mathrm{d} p_{a}}{\mathrm{~d} t}=\frac{k R T}{V_{a}} \rho Q_{a}-\frac{k p_{a}}{V_{a}} \frac{\mathrm{d} V_{a}}{\mathrm{~d} t}
$$

where $Q_{a}$ is the air flow rate in the tank, $\mathrm{m}^{3} / \mathrm{s}$.

The change in density $\rho_{a}, \mathrm{~kg} / \mathrm{m}^{3}$, the air in the tank has the form: 


$$
\frac{\mathrm{d} \rho_{a}}{\mathrm{~d} t}=\frac{1}{V_{a}} \frac{\mathrm{d} m}{\mathrm{~d} t}-\frac{m}{V_{a}^{2}} \frac{\mathrm{d} V_{a}}{\mathrm{~d} t}
$$

or

$$
\frac{\mathrm{d} \rho_{a}}{\mathrm{~d} t}=\frac{1}{V_{a}} \rho Q_{a}-\frac{\rho}{V_{a}} \frac{\mathrm{d} V_{a}}{\mathrm{~d} t}
$$

We consider the capacities of the AV and ADV as constants, that is, $\mathrm{V}_{\mathrm{a}}=\mathrm{V}_{0}==$ const. Then equations (16) and (17) take the form:

$$
\begin{aligned}
& \frac{\mathrm{d} p_{a}}{\mathrm{~d} t}=\frac{k R T}{V_{a}} \rho Q_{a} \\
& \frac{\mathrm{d} \rho_{a}}{\mathrm{~d} t}=\frac{1}{V_{a}} \rho Q_{a}
\end{aligned}
$$

We consider the capacity of the WBCh as a variable, i.e.

$$
V_{\mathrm{e}}=V_{0}+F y
$$

where $F$ is the area of the WBCh diaphragm; $y$ is displacement of the WBCh diaphragm. form:

Then, taking into account equality (20), equations (16) and (17) can be written in the

$$
\begin{aligned}
\frac{\mathrm{d} p_{a}}{\mathrm{~d} t} & =\frac{k}{V_{0}+F y}\left(\rho R T Q_{a}-p_{a} F \frac{\mathrm{d} y}{\mathrm{~d} t}\right) \\
\frac{\mathrm{d} \rho_{a}}{\mathrm{~d} t} & =\frac{\rho}{V_{0}+F y}\left(Q_{a}-F \frac{\mathrm{d} y}{\mathrm{~d} t}\right)
\end{aligned}
$$

The equation of displacement of the diaphragm, taking into account the external load $P$ (y) and the resistance $P_{s p}$ of the spring, can be represented as:

$$
m_{r m} \frac{\mathrm{d}^{2} y}{\mathrm{~d} t^{2}}=p_{a} F-P_{s p} \operatorname{sign}\left(\frac{\mathrm{d} y}{\mathrm{~d} t}\right)-P(y)
$$

where $m_{r m}$ is the reduced mass of moving parts.

We write down the resistance $P_{s p}$ of the spring in the form:

$$
P_{s p}=b \frac{\mathrm{d} y}{\mathrm{~d} t} \operatorname{sign}\left(\frac{\mathrm{d} y}{\mathrm{~d} t}\right)
$$

where $b$ is the stiffness of the spring.

The external load $P(y)$ is determined by the power characteristic of the wheel brake and is a non-linear dependence of the developed force on the movement of the brake chamber rod. Studies [21] showed that the characteristic $P(y)$ in the deceleration phase is rather 
accurately described by a polynomial of the second degree, and in the deceleration phase by a polynomial of the third degree, respectively, with the coefficients $a_{t i}$ and $a_{o t i}$ :

$$
P(y)=\left\{\begin{array}{l}
a_{\mathrm{T} 1}+a_{\mathrm{T} 2} y+a_{\mathrm{T} 3} y^{2} \\
a_{\mathrm{OT} 1}+a_{\mathrm{OT} 2} y+a_{\mathrm{OT} 3} y^{2}+a_{\mathrm{OT} 4} y^{3}
\end{array}\right.
$$

Thus, the dynamics of the AV and ADV are described by equations (18) and (19), the dynamics of the WBCh - by equations (21) - (25). Further, instead of the index " $e$ ", we use the indices of the AV, ADV, and WBCh corresponding to the elements.

Let's write down the initial and boundary conditions of the mathematical model of the pneumatic drive (see figure 1).

Initial conditions:

$$
\begin{aligned}
& Q_{\mathrm{BC}}=0, p_{\mathrm{AV}}=p_{\mathrm{ADV}}=p_{\mathrm{WBCh}}=p=p_{\mathrm{ap}}, \\
& \rho_{\mathrm{AV}}=\rho_{\mathrm{ADV}}=\rho_{\mathrm{WBCh}}=\rho=k p_{\mathrm{ap}} / a^{2}, \\
& Q_{\mathrm{AV}}=Q_{\mathrm{ADV}}=Q_{\mathrm{WBCh}}=0, y=0, \mathrm{~d} y / \mathrm{d} t=0, u=V=0 \text { or } t=0
\end{aligned}
$$

\section{Border conditions:}

\section{Plot 1}

Crane: $p_{\text {in } 1}=p_{\text {con } 1} ; p_{\text {out } 1}=p_{\text {in.p } 1} ; \rho_{\text {in } 1}=\rho_{\text {con } 1} ; \rho_{\text {out } 1}=\rho_{\text {in.p } 1}\left(p_{c}\right.$ and $\rho_{c}$ are air pressure and density in the receiver).

Pipeline $l_{1}: V_{\text {in.p } 1}=Q_{\mathrm{BC}} / f_{\mathrm{p} 1} ; p_{\text {out } 1}=p_{\mathrm{AV}} ; \rho_{\text {in } \mathrm{p} 1}=\rho_{\text {out } 1} ; \rho_{\text {out.p1 }}=\rho_{\mathrm{AV}}$.

Capacity AV: $Q_{\mathrm{AV}}=V_{\text {out.p1 }} f_{p 1}$.

\section{Plot.}

$\mathrm{AV}$ : or $p_{\mathrm{AV}}>0.15 \mathrm{MPa}$ we have $p_{\text {in } 2}=p_{\text {con } 2} ; p_{\text {out } 2}=p_{\text {in.p } 2} ; \rho_{\text {in } 2}=\rho_{\text {con } 2} ; \rho_{\text {out } 2}=\rho_{\text {in.p } 2}$ Pipeline $l_{2}: V_{\text {in.p } 2}=Q_{\mathrm{AV}} / f_{\mathrm{p} 2} ; p_{\text {out.p2 }}=p_{\mathrm{ADV}} ; \rho_{\text {in } \mathrm{p} 2}=\rho_{\text {out } 2} ; \rho_{\text {out.p } 2}=\rho_{\mathrm{ADV}}$.

Capacity ADV: $Q_{\mathrm{ADV}}=V_{\text {out.p2 }} f_{\mathrm{p} 2}$.

\section{Plot 3}

ADV: or $p_{\text {ADV }}>0,25 \mathrm{MPa}$ we have $p_{\text {in } 3}=p_{\text {con3 } 3} ; p_{\text {out } 3}=p_{\text {in.p } 3} ; \rho_{\text {in } 3}=\rho_{\text {con } 3} ; \rho_{\text {out3 }}=\rho_{\text {in.p3. }}$.

Pipeline $l_{3}: V_{\text {in.p3 }}=Q_{\mathrm{WBCh}} /\left(2 f_{\mathrm{p} 3}\right) ; p_{\text {out.p }}=p_{\mathrm{p} 4} ; \rho_{\text {in } \mathrm{p} 3}=\rho_{\text {out.p3 }} ; \rho_{\text {out.p3 }}=\rho_{\mathrm{p} 4 \text {. }}$

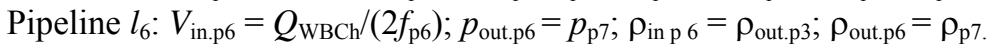

Pipeline $l_{4}: V_{\text {in.p4 }}=V_{\text {out.p3 }} / 2 ; p_{\text {out.p4 }}=p_{\mathrm{WBCh} 1} ; \rho_{\text {in } \mathrm{p} 4}=\rho_{\text {out.p3 }} ; \rho_{\text {out.p4 }}=\rho_{\mathrm{WBCh} 1 \text {. }}$

Pipeline $l_{5}: V_{\text {in.p5 }}=V_{\text {out.p }} / 2 ; p_{\text {out.p5 }}=p_{\text {WBCh } 2} ; \rho_{\text {in } 55}=\rho_{\text {out.p3 }} ; \rho_{\text {out.p5 }}=\rho_{\text {WBCh2. }}$

Pipeline $l_{7}: V_{\text {in.p7 }}=V_{\text {out.pp } 6} / 2 ; p_{\text {out.p7 }}=p_{\text {WBCh3 }} ; \rho_{\text {in p } 7}=\rho_{\text {out.p6 }} ; \rho_{\text {out.p7 }}=\rho_{\text {WBCh3. }}$.

Pipeline $l_{8}: V_{\text {in.p8 }}=\mathrm{V}_{\text {out.p6 }} / 2 ; p_{\text {out.p8 }}=p_{\mathrm{WBCh} 4} ; \rho_{\text {in } 88}=\rho_{\text {out.p6 }} ; \rho_{\text {out.p8 }}=\rho_{\mathrm{WBCh} 4 \text {. }}$

WBCh1 и WBCh2: $Q_{\text {WвCh }}=V_{\text {out.p4, } 5} f_{\mathrm{p} 4,5}$.

WBCh3 и WBCh4: $Q_{\text {WвСh }}=V_{\text {out.p7, } 8} f_{\mathrm{p} 7,8}$.

Thus, equations (1), (10) - (13), (18), (19), (21) - (25) together with the initial and boundary conditions represent a mathematical model of the considered pneumatic brake drive TT, which includes algebraic, ordinary differential equations and partial differential equations. Ordinary differential equations were solved by the Runge - Kutta method of the first order of accuracy (Euler's method).

To solve the partial differential equations, we used a modified two-layer Lax - Wendroff scheme [22], in which the calculation is carried out at half steps in space $\Delta x / 2$ and time $\Delta t / 2$ :

in the first half step by the formulas: 


$$
\begin{aligned}
& \mathrm{u}_{\mathrm{i}+1 / 2}^{\mathrm{n}+1 / 2}=\frac{1}{2}\left[u_{\mathrm{i}+1}^{\mathrm{n}}+u_{\mathrm{i}}^{\mathrm{n}}\right]-\frac{\Delta t}{2} \frac{p_{\mathrm{i}+1}^{\mathrm{n}}-p_{\mathrm{i}}^{\mathrm{n}}}{\Delta x} \\
& u_{\mathrm{i}-1 / 2}^{\mathrm{n}+1 / 2}=\frac{1}{2}\left[u_{\mathrm{i}}^{\mathrm{n}}+u_{\mathrm{i}-1}^{\mathrm{n}}\right]-\frac{\Delta t}{2} \frac{p_{\mathrm{i}}^{\mathrm{n}}-p_{\mathrm{i}-1}^{\mathrm{n}}}{\Delta x}
\end{aligned}
$$

in the second half step by the formula

$$
u_{\mathrm{i}}^{\mathrm{n}+1}=u_{\mathrm{i}}^{\mathrm{n}}-\Delta t \frac{p_{\mathrm{i}+1 / 2}^{\mathrm{n}+1 / 2}-p_{\mathrm{i}-1 / 2}^{\mathrm{n}}}{\Delta x}
$$

The stability condition for this scheme is $\Delta t / \Delta x^{2} \leq 0,5$.

A computer program in the PASKAL language was developed [23, 24].

The developed program makes it possible to solve such important tasks as determining the rational place of installation of the $\mathrm{AV}$ and the triggering time of the pneumatic drive of the brakes.

To determine the rational installation site for the AV, consider a drive limited by two sections (see figure 1). The rational place for installing the AV, at which the triggering time of the control line is minimal, is determined by calculating the change in pressure in the ADV when the lengths of the sections $l_{1}$ and $l_{2}$ change with a step of $0.1 \mathrm{~m}$ and taking into account that $l_{1}+l_{2}=$ const. The characteristics of the ADV and the AV correspond to those specified by WABCO Fahrzeugbremsen. In the calculations, the following are accepted: $d_{p 1}$ $=0.01 \mathrm{~m} ; \mathrm{d}_{\mathrm{p} 2}=0.01 \mathrm{~m} ; \mathrm{l}_{1}=0.1 \div 23.9 \mathrm{~m} ; 1_{2}=23.9 \div 0.1 \mathrm{~m} ; \mathrm{P}_{\mathrm{AV}}=0.2106 \mathrm{~Pa} ; \mathrm{V}_{\mathrm{AV}}=0.125$ $10-3 \mathrm{~m}^{3} ; \mathrm{P}_{\mathrm{ADV}}=0.45106 \mathrm{~Pa} ; \mathrm{V}_{\mathrm{ADV}}=0.2610-3 \mathrm{~m}^{3}$. The initial pressure in the system is $0.1 .106 \mathrm{~Pa}=0.1 \mathrm{MPa}$. The inlet pressure in the brake valve is $0.8106 \mathrm{~Pa}$.

\section{Results and discussion}

The results of calculating the speed of the brake drive of the ADV of the trailer through the $\mathrm{AV}$ with the total length of the control line $l=l_{1}+l_{2}=22 \mathrm{~m}$ are given below:

Time

Triggering.

\begin{tabular}{|c|c|c|c|c|c|c|c|c|c|c|}
\hline$\tau_{\text {tr. }} \mathrm{c}$ & 0.45 & 0.42 & 0.39 & 0.38 & 0.37 & 0.36 & 0.345 & 0.34 & 0.36 & 0.38 \\
\hline
\end{tabular}

Section length, m:

\begin{tabular}{|c|c|c|c|c|c|c|c|c|c|c|}
\hline$l_{1}$ & 2 & 4 & 6 & 8 & 10 & 12 & 14 & 16 & 18 & 20 \\
\hline$l_{2}$ & 20 & 18 & 16 & 14 & 12 & 10 & 8 & 6 & 4 & 2 \\
\hline
\end{tabular}

The triggering time $\tau_{t r}$ of the control line was determined from the moment the brake pedal was pressed with an interval of no more than $0.2 \mathrm{~s}$ until the achievement of $p_{A D V}=$ $0.45 \mathrm{MPa}$.

Figure 2 shows the change in the time $\tau \operatorname{tr}$ of the actuation of the control line of the brake drive of the ADV, depending on the AV location. Similarly, rational places for installing the AV in control lines of different lengths were determined.

The theoretical determination of the rational location of the AV installation in the pneumatic line of the TT brake drive, in this case with one trailer, showed good convergence with experimental studies, within 5\%. 


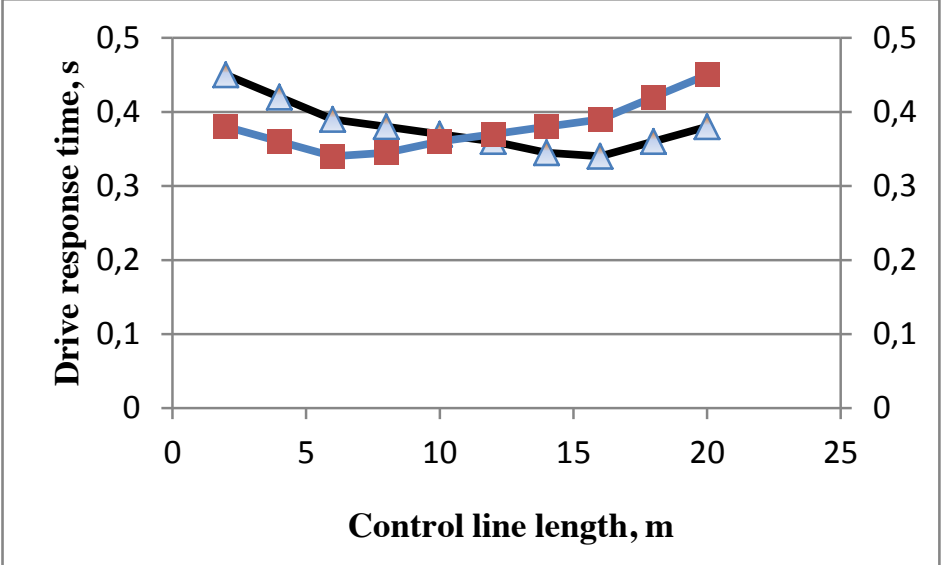

Fig. 2. Theoretical (1) and experimental (2) characteristics of the drive to determine the rational place (point) of installation of the AV in the control line of the pneumatic drive of the trailer brake.

To determine the triggering time of the pneumatic drive of the brakes, all sections of the circuit in figure 2 . In the calculation, the following parameter values were taken: $d_{\mathrm{p} 1}=d_{\mathrm{p} 2}=$ $0,012 \mathrm{~m} ; d_{\mathrm{p} 3,6}=d_{\mathrm{p} 4,5}=d_{\mathrm{p} 7,8}=0,013 \mathrm{~m} ; l_{\mathrm{p} 1}=2 \div 23,6 \mathrm{~m} ; l_{\mathrm{p} 2}=23,6 \div 2 \mathrm{~m} ; l_{\mathrm{p} 3}=3 \mathrm{~m} ; l_{\mathrm{p} 4,5}=0,6$ $\mathrm{m} ; l_{\mathrm{p} 6}=4 \mathrm{~m} ; l_{\mathrm{p} 4,5}=0,6 \mathrm{~m} ; p_{\mathrm{AV}}=0,15 \mathrm{MPa} ; p_{\mathrm{ADV}}=0,2 \mathrm{MPa} ; p_{\mathrm{WBCh}}=0,525 \mathrm{MPa} ; V_{\mathrm{AV}}=$ $0,125 \cdot 10^{-3} \mathrm{~m}^{3} ; V_{\mathrm{ADV}}=0,26 \cdot 10^{-3} \mathrm{~m}^{3} ; V_{\mathrm{WBCh} 1,2}=0,4 \cdot 10^{-3} \mathrm{~m}^{3} ; V_{\mathrm{WBCh} 3,4}=0,8 \cdot 10^{-3} \mathrm{~m}^{3}$.

The input pressure in the brake valve is $0.7 \mathrm{MPa}$.

In figure 3 as an example, the changes in pressure $p$, density $\rho$, air flow rate $Q$, and displacement $x$ of the diaphragm in the containers of the AV (section 1), ADV (section 2), and WBCh (section 3) in the trailer of a tractor (TT) are given. The calculated triggering time $\tau_{t r}=0.47 \mathrm{~s}$ is in good agreement with the experimental value of $0.46 \mathrm{~s}$.

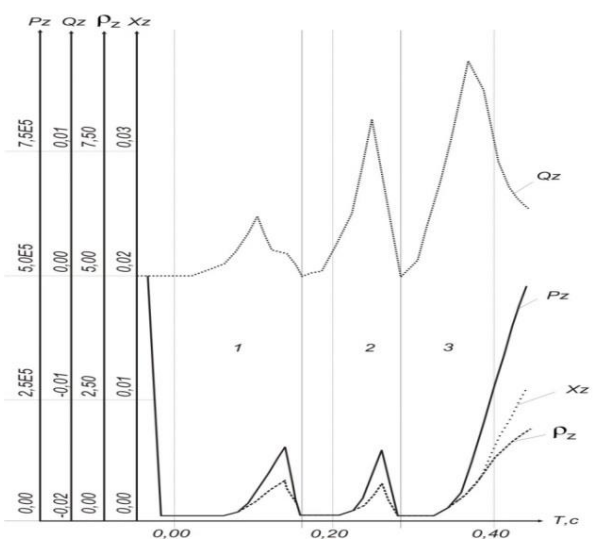

Fig. 3. Changes in the main parameters of the AV (section 1), ADV air displacement valve (section 2), and WBCh (section 3)

\section{Conclusions}

In conclusion, the study of the dynamics of the circuits of the pneumatic drive of the TT brakes showed a high convergence of the theoretical characteristics of a typical control line of a drive with an accelerating valve with experimental data. The error was no more than $5 \%$. 


\section{References}

1. Suganya S, Raja SC, Venkatesh P Simultaneous coordination of distinct plug-in hybrid electric vehicle charging stations: a modified particle swarm optimization approach. Energy 138:92-102, (2017)

2. Gao MZ, Cai GP Fault-tolerant control for wing flutter under actuator faults and time delay. J Vib Eng Technol 6(6):429-439, (2018)

3. Zhang, Z., Dong, Y. \& Han, Y. Dynamic and Control of Electric Vehicle in Regenerative Braking for Driving Safety and Energy Conservation. J. Vib. Eng. Technol. 8, 179-197, (2020).

4. R. Limpert, An investigation of the brake force distribution on tractor-semi trailer combinations, SAE Paper No. 710044, (1971)

5. R. Limpert, Brake design and safety, SAE Publication No. R-198, (1999).

6. S. Kimbrough and M. Elwell, An advanced braking and stability controller for two vehicle and trailer combinations, SAE Paper No. 931878, (1993)

7. G. Buschmann, T. Hans, and W. Kuhn, Electronic brake force distribution control-A sophisticated addition to ABS, SAE Paper No. 920646, (1992)

8. G.Yimin and M. Ehsani, Electronic braking system of EV and HEV integration of regenerative braking, automatic braking force control and ABS, SAE 2001-01-2478, (2001)

9. Salaani M, Rao S, Every J. Hardware in the loop pneumatic braking system for heavy truck testing of advanced electronic safety interventions. SAE Int J Passeng Cars: Mech Syst (2016); 9(2): 912-923.

10. Karthikeyan P, Siva Chaitanya C, Jagga Raju N, et al. Modelling an electropneumatic brake system for commercial vehicles. IET Electr Syst Transp (2011); 1(1): 41-48.

11. A.Shermukhamedov, V.Topalidi, Modeling the transport system of a multi-channel road train - Bulletin of engineering. № ISSN 0042-4633. (2014). pp 28-32.

12. E.V. Hertz, G.V. Kreinin. Calculation of pneumatic drives. - M.: Mechanical Engineering, 1975. - 272 p.

13. Roach P. Computational Fluid Dynamics. Per. from English M: Mir, (1980), -616 p.

14. Togaev A.A., Shermukhamedov A.A. Method of Calculating the Stress-Strain State of Frame Structures of the Autotractor Trailers for Static Loading Conditions // International Journal of Recent Technology and Engineering (IJRTE), ISSN: 22773878, Volume-8, Issue-5, January (2020). - pp. 4619-4623. (DOI:10.35940/ijrte.E6958.018520).

15. Osepchukov V.V., Frumkin A.K. Automobile: Structural analysis, calculation elements. $-\mathrm{M}$.: mechanical engineering, (1989). $-304 \mathrm{p}$.

16. Salimdzhanov R.T., Lebedev O.V. and others. Improving the reliability of wheeled vehicles. - T., TARI, (2007), - 338 p.

17. Parkhilovsky I.G. Automotive leaf springs. M.: Mechanical engineering, (1978). - 232 p.

18. Uspensky I.N., Melnikov A.A. Car suspension design. Moscow: Mechanical Engineering, (1976)

19. Vneshtorgizdat Ed. No. 8608T. Trailer 2PTS-4-793T. Catalog of parts and assembly units in Russian, English, French. and Spain. lang. T-Z. Order No. 971.

20. Zakin Ya.Kh. Applied theory of road train motion. M., "Mechanical Engineering", (1968)

21. Altshul A.D., Zhivotovsky L.S., Ivanov L.P. Hydraulics and Aerodynamics. - M .: Stroyizdat, (1987) . - $414 \mathrm{p}$.

22. Zakin Ya.Kh. Foundations of the theory of movement of transport and special automobile trains. Doctoral dissertation, Leningrad Agricultural Institute, (1960) 
23. Rashidov N.R. Tractor multi-link trains. -T.: "Uzbekistan", (1981). - 368 p.

24. Azizov A.A., Nishonov T. M., Meliev H. O. Mechanical-mathematical model of tractor wheel propulsor interaction with bearing surface. - ACADEMICIA: An International Multidisciplinary Research Journal № ISSN: 2249-7137. 2020. P 636644. 